\title{
Conformational Analysis of Molecular Complexes between Sodium and 1,2-Dimethoxyethane: Photoionization and ab Initio Molecular Orbital Studies
}

\author{
T.-S. Yeh and T.-M. Su* \\ Department of Chemistry, National Taiwan University, Taipei, Taiwan, R.O.C., and The Institute of the Atomic \\ and Molecular Sciences, Academia Sinica, Taipei, Taiwan, R.O.C.
}

Received: August 30, 1996; In Final Form: November 25, $1996^{\otimes}$

\begin{abstract}
The molecular complexes formed between sodium and 1,2-dimethoxyethane (DME), $\mathrm{Na}$ (DME) and $\mathrm{Na}_{2}$ (DME), were generated in a flow reactor and studied with photoionization mass spectroscopy. The photoionization threshold energies of these complexes, which are dependent on the complex conformations, were obtained from the analysis of the photoionization efficiency spectra. Ab initio molecular orbital calculations were performed on the possible conformers of these complexes and their corresponding ionic states. With the help of theoretical calculations, the photoionization threshold for the cyclic conformers of $\mathrm{Na}(\mathrm{DME})$ was determined to be $3.80 \pm 0.02 \mathrm{eV}$. For the $\mathrm{Na}_{2}$ (DME) complex, the photoionization threshold of the cyclic conformers was determined to be $4.16 \pm 0.02 \mathrm{eV}$. The conformation-dependent bond dissociation energies and the photoionization threshold energies could be rationalized and classified in terms of the sodiumLewis base bonding and the intramolecular $1,5 \mathrm{CH}_{3} / \mathrm{O}$ electrostatic interaction of 1,2-dimethoxyethane.
\end{abstract}

\section{Introduction}

There have been quite a few experimental and theoretical studies on the molecular complexes formed between the alkali metal atoms and Lewis base molecules. ${ }^{1-15}$ Most of the Lewis base molecules studied were simple molecules, such as the ammonia and water molecules. It was only very recently that the complexes between potassium and multifunctional or multidentate Lewis base molecules such as ethylenediamine and 1,2-ethanediol were reported. ${ }^{10,15}$ These complexes manifest very rich conformation structures due to the interplay between the intramolecular hydrogen bonding of the Lewis base molecules and the alkali metal atom/Lewis lone-pair electrons bonding. For these complexes, these two bonding forces happen to have similar strengths. In the present study, we extend our previous work to the 1,2-dimethoxyethane (DME) molecules, a completely different system from the above diamine and diol molecules that possesses its own unique conformational interaction energies and chemical properties.

1,2-Dimethoxyethane, besides being an important solvent, is the smallest structure subunit of poly(ethylene oxide) and cyclic crown ethers. Intensive theoretical and experimental efforts have been made on the conformational studies of this molecule. ${ }^{16-27}$ Although DME lacks the strong intramolecular hydrogen bonding that exists in 1,2-ethanediol and ethylenediamine, according to previous reports, ${ }^{19,24-27}$ there are weak 1,5 $\mathrm{CH}_{3} / \mathrm{O}$ electrostatic attractive interactions in action to stabilize some DME conformations. Energy differences among the molecular conformers tend to be smaller than those with regular intramolecular hydrogen bonding.

Up to now, there have been no reports on the properties of the complexes formed between the alkali metal atoms and the ether-related compounds such as DME. Although the alkali metal bonding strength with the ether functional group is expected to be close to or slightly weaker than that of the hydroxyl group, the conformational properties and also the chemical stabilities of these ether compounds with respect to the alkali metal are different from those of the amines and alcohols. ${ }^{10,15}$ The present study on the sodium-DME com-

\footnotetext{
${ }^{\otimes}$ Abstract published in Advance ACS Abstracts, January 15, 1997.
}

plexes form the basis for further understanding more complicated systems such as the alkali metal atoms/crown ether or poly(ethylene oxide) complexes.

In this article, the photoionization efficiency spectra and the results of molecular orbital calculations on the molecular complexes $\mathrm{Na}(\mathrm{DME})$ and $\mathrm{Na}_{2}(\mathrm{DME})$ are reported. Since the single photon ionization process connects the neutral and ionic states of the complexes, the structures and energies of the corresponding conformations of $\mathrm{Na}^{+}(\mathrm{DME})$ and $\mathrm{Na}_{2}{ }^{+}(\mathrm{DME})$ were also studied by the molecular orbital methods. The conformation-dependent ionization potentials and bond dissociation energies of these neutral and ionic complexes are emphasized in this report.

\section{Experimental Section}

The details of the experimental arrangement, which is basically composed of a photoionization mass spectrometer and a flow reactor, have been reported in previous publications. ${ }^{10,13,15}$ Only a brief account of the relevant experimental conditions for the present molecular complexes is given here. The sodium vapor was first generated by heating it in an oven to about 312 ${ }^{\circ} \mathrm{C}$ and then introduced into the flow reactor by an argon gas flow at about 0.60 Torr pressure. The temperature of the sodium flow tube was kept at $240{ }^{\circ} \mathrm{C}$. The DME vapor was directly introduced into the flow reactor through a liquid sample cell in a $40{ }^{\circ} \mathrm{C}$ water bath. During the experiments, the pressure of DME was maintained approximately at 30 mTorr and the flow reactor was fixed at $40^{\circ} \mathrm{C}$. The total pressure of the flow reactor was kept at around 2.4 Torr by an argon buffer flow.

\section{Ab Initio Molecular Orbital Calculations}

All the molecular orbital calculations were carried out by the Gaussian 94 package. ${ }^{28}$ The molecular geometries and the corresponding harmonic vibrational frequencies were calculated at the MP2(fu)/6-31G* level. All the reported energies were obtained at the level of MP2(fu)/6-311+G(d,p)//MP2(fu)/6$31 \mathrm{G}^{*}$ with a normal energy convergence of $1 \times 10^{-8}$ au. The ionization potentials were also calculated by the density functional method at the B3PW91/6-311+G(d,p)//MP2(fu)/6$31 \mathrm{G}^{*}$ level. 
TABLE 1: Conformational Energies $(\mathrm{kcal} / \mathrm{mol})$ of DME with Respect to the tTt Conformer ${ }^{a}$

\begin{tabular}{lcrrrrrrrr}
\hline & $\mathrm{tGt}$ & \multicolumn{1}{c}{$\mathrm{tGg}^{\prime}$} & $\mathrm{gGg}$ & $\mathrm{g} \mathrm{g}^{\prime} \mathrm{Tt}$ & $\mathrm{tGg}$ & $\mathrm{gGg}$ & $\mathrm{g}^{\prime} \mathrm{Gg}^{\prime}$ & $\mathrm{gTg}$ & $\mathrm{gTg}^{\prime}$ \\
\hline $\mathrm{HF}$ & 1.33 & 2.01 & 4.66 & 2.07 & 3.62 & 4.27 & 4.43 & 4.11 \\
MP2 & 0.28 & 0.39 & 1.40 & 1.55 & 1.74 & 2.05 & 2.45 & 3.16 \\
ZPEC $^{b}$ & 0.04 & -0.08 & -0.48 & -0.04 & -0.13 & -0.11 & -0.16 & -0.04 & -0.06
\end{tabular}

${ }^{a}$ Calculation level: MP2(fu)/6-311+G(d,p)//MP2(fu)/6-31G*. Energies for tTt: HF $=-307.0674798$ a.u.; MP2 $=-308.1873044$ au. ${ }^{b}$ Zeropoint energy correction with respect to the zero-point energy of $91.38 \mathrm{kcal} / \mathrm{mol}$ of the $\mathrm{tTt}$ conformer.

The conformational notations of DME are in accord with those of Radom et al. ${ }^{29}$ The symbols T (trans), G (gauche), and $\mathrm{G}^{\prime}$ (antigauche) indicate that the dihedral angle of OCCO is around $180^{\circ}, 60^{\circ}$, and $-60^{\circ}$, respectively. The lower case symbols $\mathrm{t}, \mathrm{g}$, and $\mathrm{g}^{\prime}$ denote the corresponding conformations of the COCC fragment. Since the sodium atom may also assume an eclipse position with respect to the adjacent molecular fragment, additional conformation symbols $\mathrm{e}^{0}, \mathrm{e}$, and $\mathrm{e}^{\prime}$ are used for the sodium atom if its dihedral angle $\mathrm{NaOCC}$ is within $\pm 10^{\circ}$ range of $0^{\circ},+120^{\circ}$, and $-120^{\circ}$, respectively. A full notation for a stable conformer of $\mathrm{Na}(\mathrm{DME})$ or $\mathrm{Na}^{+}(\mathrm{DME})$ would be read as $\mathrm{g}^{\prime}-\mathrm{tGt}$ or $\mathrm{Na}^{+}(\mathrm{e})-\mathrm{g}^{\prime} \mathrm{Tt}$, in which the first conformation symbol indicates the position of the sodium atom or ion, respectively, and the second one is the position of the sodiumattached methoxy group. This convention is also applied to the location of the oxygen-attached sodium atom of the disodium complexes. The vertical and adiabatic ionization potentials of the neutral complexes and the sudden and adiabatic bond dissociation energies of both the neutral and ionic complexes were calculated following the general procedure of previous publications. ${ }^{10,15}$

\section{Results and Discussion}

1. Structures and Bonding of $\mathrm{Na}(\mathrm{DME}), \mathrm{Na}_{2}(\mathrm{DME}), \mathrm{Na}^{+}$(DME), and $\mathrm{Na}_{2}{ }^{+}(\mathrm{DME})$. There are 10 stable conformers for the free 1,2-dimethoxyethane molecule in the gas phase, the same number that its alcohol counterpart, 1,2-ethanediol, has. ${ }^{30}$ Their relative conformational energies arranged in the order of the MP2 energies are shown in Table 1. Because a dominant intramolecular interaction force is lacking, the energy differences among the 10 conformers are smaller than those of 1,2ethanediol. ${ }^{30}$ With these smaller energy differences, the bonding of the sodium atom or ion to the methoxy groups becomes the dominant force in the complexes. Along this line, the structures of these sodium/DME complexes are discussed in the following.

1.1. Na(DME). Owing to the steric effect of the bulky methoxy group and the lack of dominant intramolecular interactions, the number of stable $\mathrm{Na}(\mathrm{DME})$ conformers is smaller than that of the $\mathrm{Na}(1,2$-ethanediol) complex. Seven stable conformers of $\mathrm{Na}(\mathrm{DME})$ are located by the molecular orbital methods. They could be classified into three different bonding categories: (a) a cyclic form in which sodium bonds with both methoxy groups; (b) a straight-chain form with DME in the gauche conformation in which the molecular structure is further stabilized by the electrostatic attraction between the two methoxy groups - for instance, between $\mathrm{O} 2$ and the methyl group of $\mathrm{C} 3$ in conformer g-g'Gt shown in Figure 1-and is denoted as the $1,5 \mathrm{CH}_{3} / \mathrm{O}$ intramolecular cyclic form in accordance with the convention of the literature; (c) a straight-chain form with DME in the trans conformation that is simply named as straightchain conformers in the present report. There is only one sodium complexation bond formed in the last two categories. Representative conformers for each of these three structural categories $-\mathrm{g}^{\prime}-\mathrm{tGt}, \mathrm{g}-\mathrm{g}^{\prime} \mathrm{Gt}$, and $\mathrm{e}^{0}-\mathrm{tTt}$-are shown in Figure 1.

Overall, three stable cyclic conformers $g^{\prime}-\mathrm{tGt}, \mathrm{g}^{\prime}-\mathrm{tGg}$ and $\mathrm{g}^{\prime}-$ $\mathrm{gGg}$ were located. The sodium atom for all the cyclic conformers is in the $\mathrm{g}^{\prime}$ orientation. The major geometric
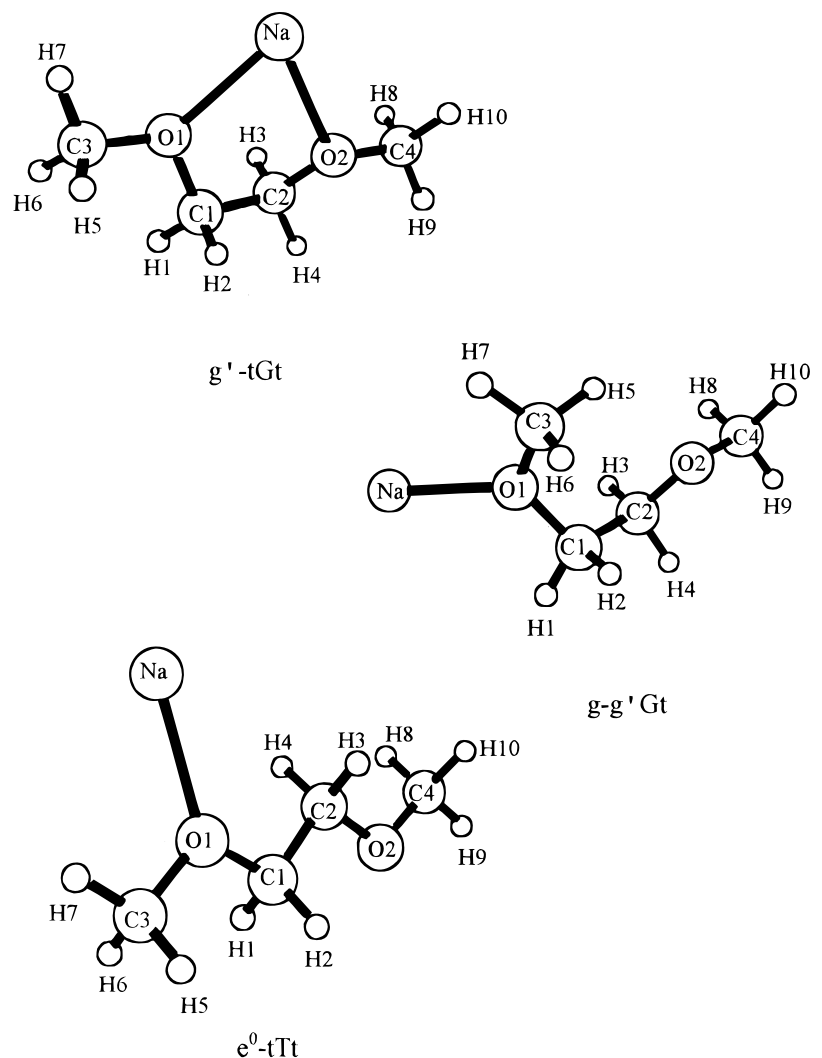

g-g' Gt

Figure 1. Schematic diagram of three representative conformers for $\mathrm{Na}(\mathrm{DME})$ : $\mathrm{g}^{\prime}$-tGt, g-g'Gt, and $\mathrm{e}^{0}$-tTt.

changes for the DME molecule in the complex formation process are the following: (1) the lengthening of all the $\mathrm{C}-\mathrm{O}$ bond lengths after the complex formation and (2) the changes of the dihedral angles of the backbone atoms to facilitate better spatial overlap of $\mathrm{Na}$ with the lone pair electrons of oxygen and to reduce steric repulsion between the methoxy groups. In the former case, for instance, the average bond length of $\mathrm{CO}$ in tGt is $1.415 \AA$, while the corresponding length is $1.426 \AA$ in $\mathrm{g}^{\prime}-\mathrm{tGt}$. For the latter case, for instance, the $\mathrm{O} 1 \mathrm{C} 1 \mathrm{C} 2 \mathrm{O} 2$ dihedral angle of the $\mathrm{g}^{\prime}-\mathrm{tGt}$ conformer changes from $71^{\circ}$ in the free conformer state to $61^{\circ}$ in the complex state. Accompanied by this shrinking of the dihedral angle is the increase of the $\mathrm{C} 3 \mathrm{O} 1 \mathrm{C} 1 \mathrm{C} 2$ and $\mathrm{C} 4 \mathrm{O} 2 \mathrm{C} 2 \mathrm{C} 1$ dihedral angles to minimize steric repulsion between the methoxy groups. For the $\mathrm{g}^{\prime}-\mathrm{tGg}$ conformer, the $\mathrm{C} 4 \mathrm{O} 2 \mathrm{C} 2 \mathrm{C} 1$ dihedral angle changes from $62^{\circ}$ in the free conformer state to $85^{\circ}$ in the complex while the $\mathrm{O} 1 \mathrm{C} 1 \mathrm{C} 2 \mathrm{O} 2$ and the $\mathrm{C} 3 \mathrm{O} 1 \mathrm{C} 1 \mathrm{C} 2$ dihedral angles do not change appreciably.

In the conformations with the $1,5 \mathrm{CH}_{3} / \mathrm{O}$ intramolecular cyclic structure, only g-g'Gt and e-g'Gg conformers were found even though there are actually more choices of the Lewis electron lone-pairs that could be used as bonding sites for sodium than those of the above cyclic form. In these conformers, $\mathrm{Na}$ simultaneously bonds with the two electron lone pairs of $\mathrm{O} 1$ of the first methoxy group. This forms a more stable sodium bond. If $\mathrm{Na}$ is bonded to $\mathrm{O} 2$ of the second methoxy group, the 1,5 $\mathrm{CH}_{3} / \mathrm{O}$ electrostatic attraction would weaken and the internal cyclic structure become unstable. Only those conformers with 

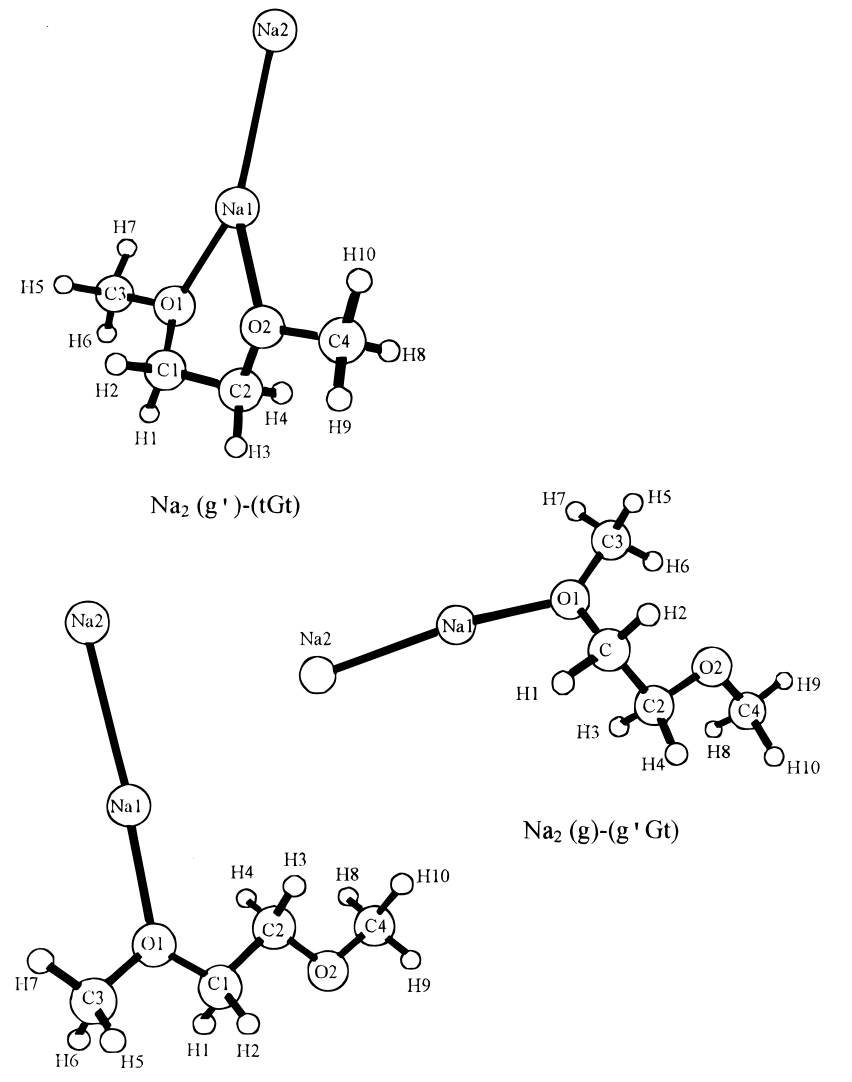

$\mathrm{Na}_{2}(\mathrm{~g})-\left(\mathrm{g}^{\prime} \mathrm{Gt}\right)$

$$
\mathrm{Na}_{2}\left(\mathrm{e}^{0}\right)-(\mathrm{tTt})
$$

Figure 2. Schematic diagram of three representative conformers for $\mathrm{Na}_{2}(\mathrm{DME}): \mathrm{Na}_{2}\left(\mathrm{~g}^{\prime}\right)-\mathrm{tGt}, \mathrm{Na}_{2}(\mathrm{~g})-\mathrm{g}^{\prime} \mathrm{Gt}$ and $\mathrm{Na}_{2}\left(\mathrm{e}^{0}\right)-\mathrm{tTt}$.

$\mathrm{Na}$ bonded to $\mathrm{O} 1$ are formed for this class of conformation. Finally, three straight-chain conformers g-g'Tt, $\mathrm{e}^{0}-\mathrm{tTt}$, and $\mathrm{g}^{\prime}-$ $\mathrm{tTg}^{\prime}$ were obtained. For these monodentate conformers, the $\mathrm{Na}-\mathrm{O}$ bond lengths are all shorter than those with bidentate bonding by about $0.07 \AA$. Their remaining primary geometries are all quite close to each other.

1.2. $\mathrm{Na}_{2}(\mathrm{DME})$. Being similar to the monosodium complex, the stable conformers of $\mathrm{Na}_{2}$ (DME) also could be classified into three conformational categories: (1) cyclic conformers in which $\mathrm{Na}_{2}$ bonds with both methoxy groups; (2) straight-chain form with DME in gauche conformation in which $\mathrm{Na}_{2}$ bonds with only one methoxy group of DME, which has an intramolecular cyclic structure via $1,5 \quad \mathrm{CH}_{3} / \mathrm{O}$ electrostatic attraction; (3) straight-chain form with DME in the trans conformation. For all the possible DME conformers, the two sodium atoms could act as a unit in forming a sodium bond or they bond to the two methoxy groups independently. It was found that the former cases are always more stable, and therefore, they are considered in this report. Figure 2 shows three representative conformers of $\mathrm{Na}_{2}(\mathrm{DME})$ for each of the three conformational categories: $\mathrm{Na}_{2}\left(\mathrm{~g}^{\prime}\right)-\mathrm{tGt}, \mathrm{Na}_{2}(\mathrm{~g})-\mathrm{g}^{\prime} \mathrm{Gt}$, and $\mathrm{Na}_{2}\left(\mathrm{e}^{0}\right)$-tTt. Three stable conformers were located for the first group and two for the second group. For the straight-chain conformers, only the representative conformer $\mathrm{Na}_{2}\left(\mathrm{e}^{0}\right)$-tTt was considered here.

In general, the geometric parameters of the disodium complex are similar to those of the corresponding monosodium systems except for the $\mathrm{Na}-\mathrm{O}$ bond distance. Owing to a stronger interaction strength, the $\mathrm{Na}-\mathrm{O}$ bond distances of the disodium complex are shorter than those of the monosodium complex by about $0.04 \AA$ for the cyclic form. The $\mathrm{Na}-\mathrm{Na}$ bond length in the complex is longer than the free $\mathrm{Na}_{2}$ bond length by $0.09 \AA$. For the other conformational groups, the corresponding lengthening is only $0.04 \AA$. Apparently, the bidentate interaction of

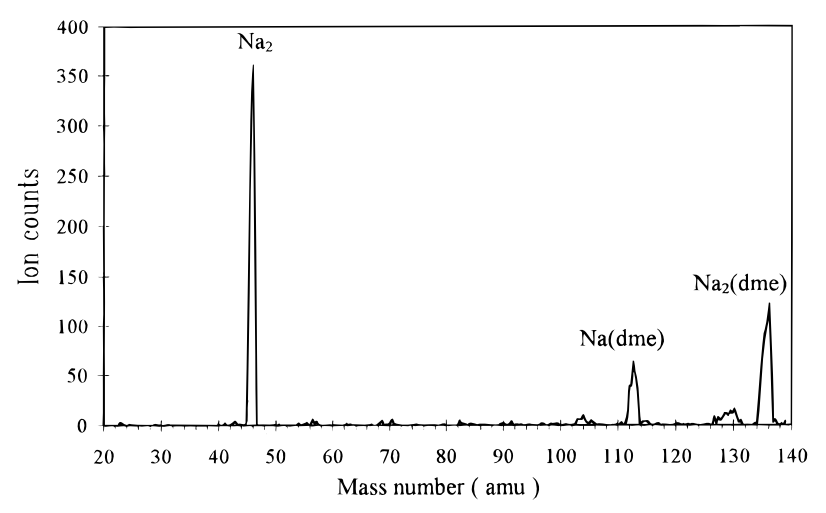

Figure 3. Photoionization mass spectrum of $\mathrm{Na}(\mathrm{DME})$ and $\mathrm{Na}_{2}(\mathrm{DME})$ at $255 \mathrm{~nm}$ laser radiation.

the cyclic conformers induces about twice the bond lengthening of that of the monodentate conformer.

1.3. $\mathrm{Na}^{+}(\mathrm{DME})$ and $\mathrm{Na}_{2}{ }^{+}(\mathrm{DME})$. Only five stable $\mathrm{Na}^{+}(\mathrm{DME})$ conformers were located. Two of them are the cyclic conformers: $\mathrm{Na}^{+}\left(\mathrm{g}^{\prime}\right)-\mathrm{tGt}$ and $\mathrm{Na}^{+}\left(\mathrm{g}^{\prime}\right)-\mathrm{tGg}$. The other three are all in straight-chain form: $\mathrm{Na}^{+}(\mathrm{e})-\mathrm{g}^{\prime} \mathrm{Tt}, \mathrm{Na}^{+}\left(\mathrm{e}^{0}\right)-\mathrm{tTt}$, and $\mathrm{Na}^{+}\left(\mathrm{e}^{0}\right)-\mathrm{tTg}^{\prime}$. No stable ionic conformers with intramolecular $1,5 \mathrm{CH}_{3} / \mathrm{O}$ interaction were found. The strong ion-dipole interaction forces all the members of this group to take a cyclic conformer in the ionic state. For these conformers, the $\mathrm{NaO} 1$ bond length is shortened by an average of $0.177 \AA$ compared to those of the corresponding neutral complex. On the other hand, all the $\mathrm{C}-\mathrm{O}$ lengths increase compared to those of the neutral complex.

For $\mathrm{Na}_{2}{ }^{+}(\mathrm{DME})$, an exhaustive search for all the possible stable conformers was not attempted. Only those related to the photoionization study were calculated and reported here. Compared to the corresponding neutral conformers, the $\mathrm{Na} 1 \mathrm{O}$ bond lengths of $\mathrm{Na}_{2}{ }^{+}\left(\mathrm{g}^{\prime}\right)-\mathrm{tGt}$ and $\mathrm{Na}_{2}{ }^{+}\left(\mathrm{g}^{\prime}\right)-\mathrm{tGg}$ are shorter by 0.10 and $0.095 \AA$, respectively. Owing to the stronger cationic bonding, this is an expected consequence. In general, the $\mathrm{Na}_{2}{ }^{+}$ bond length in the complexes is longer than that of the free $\mathrm{Na}_{2}{ }^{+}$molecule, which in turn is much longer than that of the neutral sodium molecule. For instance, the $\mathrm{Na}_{2}{ }^{+}$bond length of $\mathrm{Na}_{2}{ }^{+}\left(\mathrm{g}^{\prime}\right)-\mathrm{tGt}$ is longer than those of the free $\mathrm{Na}_{2}{ }^{+}$by 0.13 $\AA$, and for the $\mathrm{Na}_{2}{ }^{+}\left(\mathrm{g}^{\prime}\right)-\mathrm{tGg}$ complex, it is longer by $0.06 \AA$. Note that at the present calculation level, the $\mathrm{Na}_{2}{ }^{+}$bond length is $0.50 \AA$ longer than the $\mathrm{Na}_{2}$ bond length.

The geometric parameters and the harmonic vibrational frequencies of the above neutral and ionic complexes are tabulated in the Supporting Information.

2. Photoionization Mass Spectra and Photoionization Efficiency Spectra of $\mathrm{Na}(\mathrm{DME})$ and $\mathrm{Na}_{2}(\mathrm{DME})$. Figure 3 shows the photoionization mass spectrum of $\mathrm{Na}(\mathrm{DME})$ and $\mathrm{Na}_{2}$ (DME) at $255 \mathrm{~nm}$ radiation. Turning off either the 1,2dimethoxyethane vapor or the sodium vapor caused the signals of the sodium complexes to disappear. Together with the atomic mass unit of the signals, this suggests that the expected sodium complexes were generated and photoionized by the laser radiation. The signal of $\mathrm{Na}_{2}(\mathrm{DME})$ is stronger than that of $\mathrm{Na}(\mathrm{DME})$ under the present experimental conditions.

Figure 4 shows the photoionization efficiency spectrum of $\mathrm{Na}_{2}$ (DME) and its corresponding Watanabe plot. The spectrum clearly reveals a step-function-like rising in the longer wavelength region and a slow decrease of the signal beyond 35400 $\mathrm{cm}^{-1}$. A possible dissociation of the photoionized $\mathrm{Na}_{2}(\mathrm{DME})$ complexes into the monosodium complex as the photon energy reaches this wavelength range may account for this decrease of the signal. The photoionization threshold of $\mathrm{Na}_{2}(\mathrm{DME})$ is determined to be $4.16 \pm 0.02 \mathrm{eV}$ from the crossing point of two least-squares fitted lines in the Watanabe plot shown in 

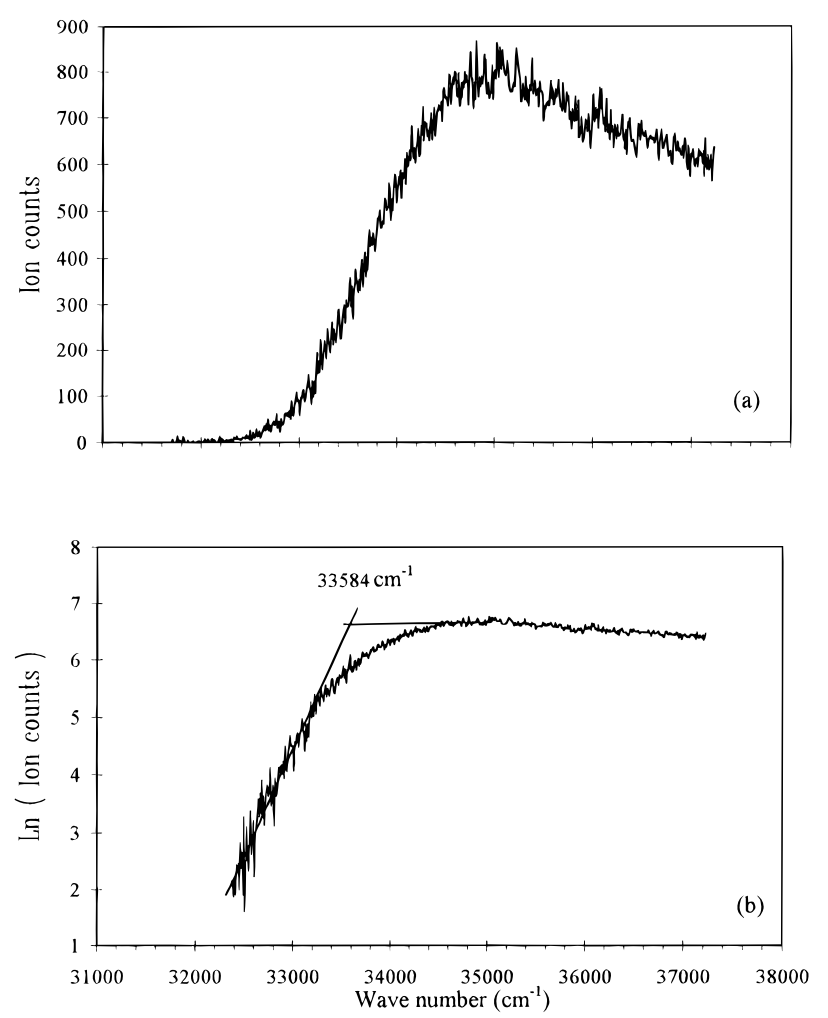

Figure 4. Photoionization efficiency spectrum of $\mathrm{Na}_{2}$ (DME) (a) and the corresponding Watanabe plot (b).

Figure 4b. The uncertainty was estimated from the other possible different selections of the linear regions in the spectrum for the Watanabe plot. The threshold ionization potentials of $\mathrm{Na}$ and $\mathrm{Na}_{2}$ were measured to be 5.134 and $4.875 \mathrm{eV}$ by the present experimental setup. Compared with the corresponding reported adiabatic ionization potentials of $5.139^{31}$ and 4.895 $\mathrm{eV},{ }^{32}$ respectively, the agreement is good.

Figure 5 shows the photoionization efficiency spectrum and its corresponding Watanabe plot of $\mathrm{Na}(\mathrm{DME})$. Two threshold steps are distinguishable in the spectrum. The first threshold appears around $31000 \mathrm{~cm}^{-1}$. From the crossing point of the two least-squares lines in the Watanabe plot, the first threshold ionization energy is determined to be $3.80 \pm 0.02 \mathrm{eV}$. After the first threshold point, the ion signal rises slowly until it reaches the second threshold at around $35000 \mathrm{~cm}^{-1}$. By use of the same procedure, the second threshold energy is determined to be $4.33 \pm 0.02 \mathrm{eV}$. As the photon energy reaches $35400 \mathrm{~cm}^{-1}$, the signal starts another slow rise to the higherenergy region. This photon energy range is consistent with that for the decrease of the above photoionization signal of $\mathrm{Na}_{2}(\mathrm{DME})$. The observation further supports the possible dissociation of the photoionized cationic disodium complex into the monosodium species in this photon region.

3. Comparison between Theoretical and Experimental Results. The theoretical vertical and adiabatic ionization potentials are listed in Table 2. Two types of adiabatic ionization potentials are calculated: one is the adiabatic ionization potential with respect to the most stable ionic state conformer $\mathrm{Na}^{+}-\mathrm{tGt}$ and another is the potential with respect to its initial conformation state. The difference between the vertical and the second type of adiabatic ionization potentials is an indicator of the geometric variations between the neutral and ionic states. By comparison of the present experimental threshold ionization energies with the theoretical vertical ionization potentials, the photoionization efficiency spectra could be assigned as the contributions of the various conformers.
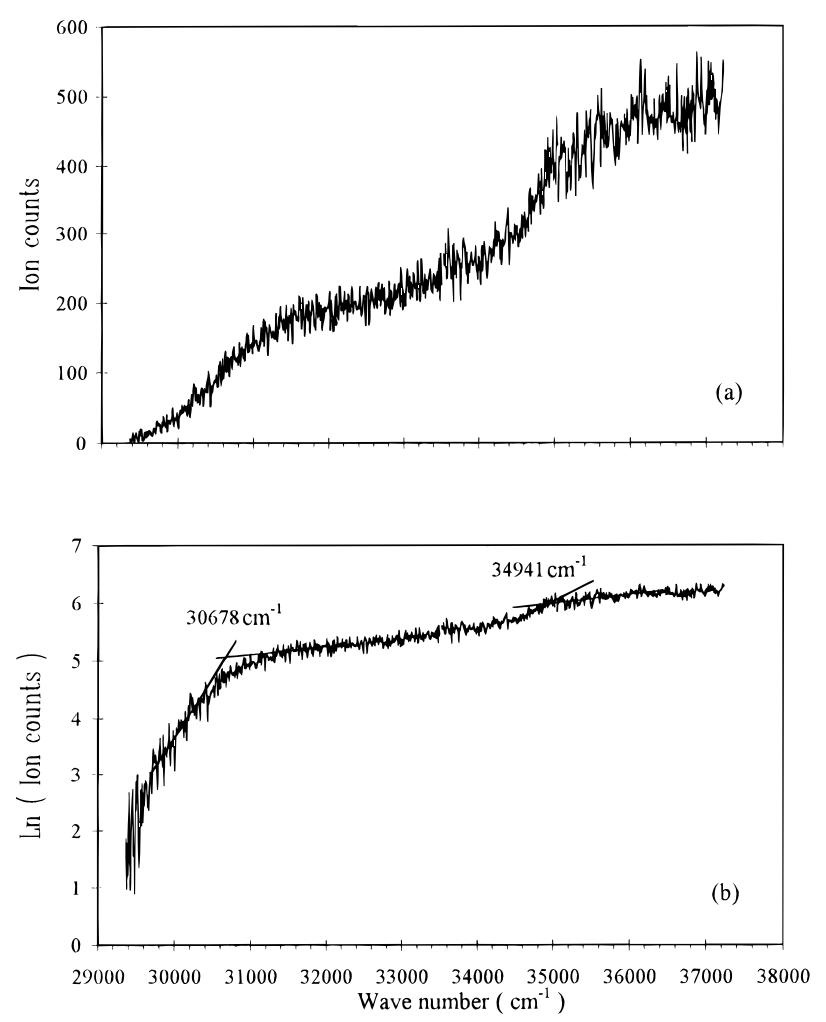

Figure 5. Photoionization efficiency spectrum of $\mathrm{Na}(\mathrm{DME})$ (a) and the corresponding Watanabe plot (b).

As shown in Table 2, ionization potentials were calculated at three levels: HF, MP2, and B3PW91. Comparing with the experimental adiabatic ionization potentials of $\mathrm{Na}$ and $\mathrm{Na}_{2}, 5.139$ $\mathrm{eV}^{31}$ and $4.895 \mathrm{eV},{ }^{32}$ one finds that the HF method consistently underestimates the ionization energies; the MP2 method yields a better value for the sodium atoms yet still off by $0.39 \mathrm{eV}$ for the sodium molecules; the B3PW91 method agrees with the experimental $\mathrm{Na}$ ionization potential to $0.13 \mathrm{eV}$ and that of $\mathrm{Na}_{2}$ to $0.02 \mathrm{eV}$. This is in consistence with the recent assessment of the B3PW91 method in the prediction of the ionization potentials. ${ }^{33}$ In the following discussion, the B3PW91 ionization potentials shall be adapted for the comparison with the experimental results.

As shown in Table 2, there are always some minor deviations between the vertical ionization potentials and the adiabatic ionization potentials with respect to its own conformation state. It ranges from around $0.11 \mathrm{eV}$ for the cyclic conformers of the mono- and disodium complexes to around $0.05 \mathrm{eV}$ for the straight-chain conformers of the monosodium complexes. The $\mathrm{Na}_{2}$ molecule also has a large $0.09 \mathrm{eV}$ difference for these two ionization potentials. As expected, the cyclic conformers, whose sodium ions are fully bonded with the bidentate DME after being photoionized, undergo more significant geometric changes than monodentate conformers. These are expected, since the bidentate interactions are always much stronger than those of the corresponding monodentate state.

For both the mono- and disodium complexes, the theoretical vertical ionization potentials of the conformers could be grouped into two energy clusters: (1) for $\mathrm{Na}(\mathrm{DME})$, the average ionization potential of the cyclic conformers is $3.83 \mathrm{eV}$, and that of the remaining conformers is $4.37 \mathrm{eV}$; (2) for $\mathrm{Na}_{2}(\mathrm{DME})$, the average ionization potential of the cyclic conformers is 4.11 $\mathrm{eV}$, and that of the remaining conformers is $4.40 \mathrm{eV}$.

The experimental photoionization threshold for $\mathrm{Na}_{2}$ (DME) is $4.16 \mathrm{eV}$. Compared with the average theoretical vertical ionization potential of the cyclic conformers of $4.11 \mathrm{eV}$, the 
TABLE 2: Theoretical and Experimental Ionization Potentials $(\mathrm{eV})$ of $\mathrm{Na}(\mathrm{DME})$ and $\mathrm{Na}_{2}(\mathrm{DME})^{a}$

\begin{tabular}{|c|c|c|c|c|c|c|c|c|c|c|c|}
\hline & \multirow[b]{2}{*}{ form $^{b}$} & \multicolumn{3}{|c|}{ vertical } & \multicolumn{6}{|c|}{ adiabatic } & \multirow[b]{2}{*}{ exptl } \\
\hline & & $\mathrm{HF}$ & MP2 & B3PW91 & $\mathrm{HF}^{c}$ & $\mathrm{MP} 2^{c}$ & B3PW91 ${ }^{c}$ & $\mathrm{HF}^{d}$ & $\mathrm{MP}^{d}$ & $\mathrm{~B} 3 \mathrm{PW}{ }^{d}$ & \\
\hline $\mathrm{Na} \rightarrow \mathrm{Na}^{+}$ & & & & & 4.94 & 5.00 & 5.27 & & & & $5.139^{e}$ \\
\hline $\mathrm{Na} 2 \rightarrow \mathrm{Na}_{2}^{+}$ & & 4.10 & 4.61 & 4.97 & 3.98 & 4.51 & 4.88 & & & & $4.895^{f}$ \\
\hline $\mathrm{g}^{\prime} \mathrm{tGGt} \rightarrow \mathrm{Na}^{+}-\mathrm{DME}^{g}$ & cy & 3.43 & 3.60 & 3.83 & 3.33 & 3.49 & 3.72 & 3.33 & 3.49 & 3.72 & 3.80 \\
\hline $\mathrm{g}^{\prime} \mathrm{tGg} \rightarrow \mathrm{Na}^{+}-\mathrm{DME}$ & cy & 3.42 & 3.59 & 3.82 & 3.24 & 3.42 & 3.66 & 3.31 & 3.49 & 3.72 & \\
\hline $\mathrm{g}^{\prime}-\mathrm{gGg} \rightarrow \mathrm{Na}^{+}-\mathrm{DME}$ & cy & 3.43 & 3.60 & 3.84 & 3.11 & 3.31 & 3.55 & $h$ & $h$ & $h$ & \\
\hline $\mathrm{g}-\mathrm{g}^{\prime} \mathrm{Gt} \rightarrow \mathrm{Na}^{+}-\mathrm{DME}$ & in & 3.93 & 4.07 & 4.32 & 3.18 & 3.33 & 3.61 & $h$ & $h$ & $h$ & \\
\hline e-g' $\mathrm{Gg} \rightarrow \mathrm{Na}^{+}-\mathrm{DME}$ & in & 3.95 & 4.08 & 4.33 & 3.07 & 3.25 & 3.52 & $h$ & $h$ & $h$ & \\
\hline $\mathrm{g}-\mathrm{g}^{\prime} \mathrm{Tt} \rightarrow \mathrm{Na}^{+}-\mathrm{DME}$ & st & 3.98 & 4.11 & 4.37 & 3.15 & 3.26 & 3.56 & 3.94 & 4.06 & 4.32 & \\
\hline $\mathrm{e}^{0}-\mathrm{tTt} \rightarrow \mathrm{Na}^{+}-\mathrm{DME}$ & st & 4.00 & 4.13 & 4.39 & 3.17 & 3.28 & 3.58 & 3.94 & 4.07 & 4.32 & \\
\hline $\mathrm{g}^{\prime}-\mathrm{tTg}^{\prime} \rightarrow \mathrm{Na}^{+}-\mathrm{DME}$ & st & 4.02 & 4.15 & 4.40 & 3.07 & 3.19 & 3.49 & 3.93 & 4.06 & 4.31 & \\
\hline $\mathrm{Na}_{2}\left(\mathrm{~g}^{\prime}\right)-\mathrm{tGt} \rightarrow \mathrm{Na}_{2}{ }^{+}-\mathrm{DME}$ & cy & 3.21 & 3.81 & 4.11 & 3.08 & 3.70 & 4.01 & 3.08 & 3.70 & 4.01 & 4.16 \\
\hline $\mathrm{Na}_{2}\left(\mathrm{~g}^{\prime}\right)-\mathrm{tGg} \rightarrow \mathrm{Na}_{2}^{+}-\mathrm{DME}$ & cy & 3.22 & 3.82 & 4.12 & 2.99 & 3.62 & 3.94 & 3.06 & 3.69 & 4.00 & \\
\hline $\mathrm{Na}_{2}\left(\mathrm{~g}^{\prime}\right)-\mathrm{gGg} \rightarrow \mathrm{Na}_{2}{ }^{+}-\mathrm{DME}$ & cy & 3.20 & 3.80 & 4.10 & 2.86 & 3.50 & 3.83 & $h$ & $h$ & $h$ & \\
\hline $\mathrm{Na}_{2}(\mathrm{~g})-\mathrm{g}^{\prime} \mathrm{Gt} \rightarrow \mathrm{Na}_{2}{ }^{+}-\mathrm{DME}$ & in & 3.52 & 4.08 & 4.40 & 2.86 & 3.45 & 3.83 & $h$ & $h$ & $h$ & \\
\hline $\mathrm{Na}_{2}(\mathrm{~g})-\mathrm{g}^{\prime} \mathrm{Gg} \rightarrow \mathrm{Na}_{2}{ }^{+}-\mathrm{DME}$ & in & 3.52 & 4.08 & 4.40 & 2.74 & 3.36 & 3.74 & $h$ & $h$ & $h$ & \\
\hline $\mathrm{Na}_{2}\left(\mathrm{e}^{0}\right)-\mathrm{tTt} \rightarrow \mathrm{Na}_{2}^{+}-\mathrm{DME}$ & st & 3.57 & 4.14 & 4.46 & 2.85 & 3.40 & 3.80 & 3.45 & 4.03 & 4.37 & \\
\hline
\end{tabular}

${ }^{a}$ Calculation levels: MP2(fu)/6-311+G(d,p)// MP2(fu)/6-31G* and B3PW91/6-311+G(d,p)// MP2(fu)/6-31G*. ${ }^{b}$ Complex conformations: cy, cyclic; in, $1,5 \mathrm{CH}_{3} / \mathrm{O}$ intramolecular interaction; st, straight chain. ${ }^{c}$ Adiabatic ionization potential with respect to the most stable conformer Na ${ }^{+}\left(\mathrm{g}^{\prime}\right)$ tGt. ${ }^{d}$ Adiabatic ionization potential with respect to its own conformation state. ${ }^{\mathrm{e}}$ Reference $31 .{ }^{f}$ Adiabatic ionization potential by ref $32 .{ }^{g}$ DME: 1,2-dimethoxyethane. ${ }^{h}$ Parent neutral conformation is not locally stable in ionic form.

agreement is good. The ionization spectrum of the disodium complex starts to decrease as the photon energy increases over $35400 \mathrm{~cm}^{-1}$. The signature of the second ionization energy cluster, which is supposed to be due to the remaining conformers and is predicted to be around $4.40 \mathrm{eV}$, is not observed in the present experiment.

The first experimental threshold for $\mathrm{Na}(\mathrm{DME})$ appears at 3.80 $\pm 0.02 \mathrm{eV}$. The theoretical vertical ionization potential for the cyclic conformation group is $3.83 \mathrm{eV}$. The agreement is excellent. The second experimental threshold, which is assigned as the second step-function-like feature in the photoionization efficiency spectrum, appears at $4.33 \mathrm{eV}$. Theoretically, the average vertical ionization energy of the second ionization energy cluster-g-g'Gt, e-g'Gg, g-g'Tt, e ${ }^{0}-\mathrm{tTt}, \mathrm{g}^{\prime}-\mathrm{tTg}^{\prime}$ - is 4.37 $\mathrm{eV}$. The excellent agreement may suggest that the second measured ionization threshold is solely due to the contribution of the monosodium complex. However, compared to the spectral shape of the first threshold, the long slowly rising slope before the second threshold suggests that a number of conformers with slightly different ionization threshold potentials are contributing to this part of the spectrum. It is also known theoretically that the second ionization threshold of the disodium complex of $4.40 \mathrm{eV}$ is in the same ionization region as the second monosodium complex and that their photogenerated ionic state possesses a high enough internal energy to dissociate into the monosodium ionic fragments as indicated in the disodium complex spectrum. The present experimental and theoretical data could not rule out either one of the above two pathways in the second ionization threshold energy region. Nevertheless, the long tail at the lower ionization energy seems to suggest the presence of the second energy cluster of the monosodium complex, which has a comparatively lower theoretical ionization threshold energy at $4.32 \mathrm{eV}$, and the decay of the disodium complex signal as the photon energy goes beyond $4.34 \mathrm{eV}$ suggests the possible contribution of the monosodium ions due to the disodium dissociation channel in the higher-energy region. It is therefore suggested that the second ionization threshold of the monosodium complex spectrum is the combined signature of the above two ionization pathways. We shall discuss the details of the energetic considerations in the following subsection.

4. Bond Dissociation Energies of $\mathrm{Na}(\mathrm{DME}), \mathrm{Na}_{2}$ (DME), $\mathrm{Na}^{+}$(DME), and $\mathrm{Na}_{2}{ }^{+}$(DME). Two types of bond dissociation energies were calculated. The sudden bond dissociation energy is the dissociation energy with the molecular fragments retaining their original geometries in the parent molecule. The adiabatic bond dissociation energy is the dissociation energy with the molecular fragments relaxing to their final energy minimum states. The former quantity is served as a measure of the interaction strength between sodium and DME. The latter quantity is the thermal stability of the conformer at $0 \mathrm{~K}$. Tables 3 and 4 list the bond dissociation energies calculated at the MP2(full)/6-311+G(d,p)//MP2(full)/6-31G* level for $\mathrm{Na}(\mathrm{DME})$ and $\mathrm{Na}_{2}$ (DME), and their corresponding ionic complexes, respectively.

As mentioned earlier, there are two major interaction forces in the sodium complexes: the sodium bonding and the 1,5 $\mathrm{CH}_{3} / \mathrm{O}$ electrostatic attractive interaction. The sodium bonding is stronger than the $1,5 \mathrm{CH}_{3} / \mathrm{O}$ attractive interaction and is the dominant factor for the stability of the complexes. Two forms of the sodium bonding are possible: the bidentate interaction and the monodentate interaction. For monosodium complex, the $g^{\prime}-\mathrm{tGt}, \mathrm{g}^{\prime}-\mathrm{tGg}$, and $\mathrm{g}^{\prime}-\mathrm{gGg}$ cyclic conformers have the bidentate type sodium bonding interaction and are the most stable complexes. The remaining conformers belong to the monodentate type sodium bonding interaction. As shown in the first two columns of Table 3 , the sudden dissociation energies of the cyclic conformers are approximately twice the value of those of the monodentate conformers. Among the monodentate conformers, there is still a minor difference between the straight-chain conformers and the conformers with $1,5 \mathrm{CH}_{3} / \mathrm{O}$ interactions. The sudden dissociation energies of the former type are always slightly less than those of the latter category. The $1,5 \mathrm{CH}_{3} / \mathrm{O}$ interaction enhances the bonding strength of the sodium-DME bond. A similar trend is also observed previously in the complexes with intramolecular hydrogen bonding such as potassium-1,2-ethanediol. ${ }^{15}$ The above observations are also true for the disodium complex listed in the same table.

The middle three columns of Table 3 list the adiabatic equilibrium dissociation energies and the zero-point energy corrections with respect to the most stable conformer tTt. As expected, the bidentate conformers are more stable than the monodentate conformers for both mono- and disodium complexes. Among the conformers of the same bonding category, their relative energy stabilities are generally determined by the relative stabilities of the corresponding DME conformers. For instance, in the bidentate bonding category, $\mathrm{g}^{\prime}-\mathrm{tGt}$ is more stable 
TABLE 3: Theoretical Dissociation Energies (kcal/mol) of Na(DME) and $\mathrm{Na}_{2}(\mathrm{DME})(\mathrm{MP2}(\mathrm{fu}) / 6-311+G(\mathrm{~d}, \mathrm{p}) / / \mathrm{Mp} 2(\mathrm{fu}) / 6-31 G *)$

\begin{tabular}{|c|c|c|c|c|c|c|c|c|}
\hline & \multicolumn{2}{|c|}{ sudden } & \multicolumn{6}{|c|}{ adiabatic } \\
\hline & $\mathrm{HF}$ & MP2 & $\mathrm{HF}^{a}$ & $\mathrm{MP}^{a}$ & $\mathrm{ZPEC}^{b}$ & $\mathrm{HF}^{c}$ & $\mathrm{MP} 2^{c}$ & $\mathrm{ZPEC}^{d}$ \\
\hline $\mathrm{g}^{\prime} \mathrm{t} \mathrm{Gt} \rightarrow \mathrm{Na}+\mathrm{DME}^{e}$ & 9.02 & 11.03 & 6.05 & 9.63 & -0.80 & 7.38 & 9.91 & -0.84 \\
\hline $\mathrm{g}^{\prime}-\mathrm{tGg} \rightarrow \mathrm{Na}+\mathrm{DME}$ & 8.65 & 10.80 & 4.01 & 7.92 & -0.77 & 7.63 & 9.65 & -0.65 \\
\hline $\mathrm{g}^{\prime}-\mathrm{gGg} \rightarrow \mathrm{Na}+\mathrm{DME}$ & 7.63 & 10.02 & 0.97 & 5.32 & -0.50 & 5.63 & 6.72 & -0.43 \\
\hline $\mathrm{g}-\mathrm{g}^{\prime} \mathrm{Gt} \rightarrow \mathrm{Na}+\mathrm{DME}$ & 5.07 & 6.58 & 2.60 & 5.94 & -0.55 & 4.62 & 6.33 & -0.47 \\
\hline $\mathrm{e}-\mathrm{g}^{\prime} \mathrm{Gg} \rightarrow \mathrm{Na}+\mathrm{DME}$ & 4.79 & 6.34 & 0.03 & 4.02 & -0.57 & 4.30 & 6.07 & -0.46 \\
\hline $\mathrm{g}-\mathrm{g}^{\prime} \mathrm{Tt} \rightarrow \mathrm{Na}+\mathrm{DME}$ & 4.40 & 6.04 & 1.87 & 4.24 & -0.49 & 3.94 & 5.78 & -0.45 \\
\hline $\mathrm{e} 0-\mathrm{tTt} \rightarrow \mathrm{Na}+\mathrm{DME}$ & 2.93 & 4.85 & 2.37 & 4.58 & -0.45 & 2.37 & 4.58 & -0.45 \\
\hline $\mathrm{g}^{\prime}-\mathrm{tTg}^{\prime} \rightarrow \mathrm{Na}+\mathrm{DME}$ & 2.60 & 4.55 & -0.03 & 2.68 & -0.03 & 2.04 & 4.23 & -0.42 \\
\hline $\mathrm{Na}_{2}\left(\mathrm{~g}^{\prime}\right)-\mathrm{tGt} \rightarrow \mathrm{Na}_{2}+\mathrm{DME}$ & 15.10 & 17.59 & 12.09 & 15.96 & -0.92 & 13.42 & 16.24 & -0.96 \\
\hline $\mathrm{Na}_{2}\left(\mathrm{~g}^{\prime}\right)-\mathrm{tGg} \rightarrow \mathrm{Na}_{2}+\mathrm{DME}$ & 14.60 & 17.35 & 9.83 & 14.20 & -0.91 & 13.46 & 15.93 & -0.78 \\
\hline $\mathrm{Na}_{2}\left(\mathrm{~g}^{\prime}\right)-\mathrm{gGg} \rightarrow \mathrm{Na}_{2}+\mathrm{DME}$ & 13.54 & 16.36 & 6.82 & 11.44 & -0.87 & 11.48 & 12.85 & -0.39 \\
\hline $\mathrm{Na}_{2}(\mathrm{~g})-\mathrm{g}^{\prime} \mathrm{Gt} \rightarrow \mathrm{Na}_{2}+\mathrm{DME}$ & 9.38 & 10.97 & 6.92 & 10.25 & -0.63 & 8.93 & 10.64 & -0.55 \\
\hline $\mathrm{Na}_{2}(\mathrm{~g})-\mathrm{g}^{\prime} \mathrm{Gg} \rightarrow \mathrm{Na}_{2}+\mathrm{DME}$ & 9.04 & 10.62 & 4.27 & 8.21 & -0.64 & 8.54 & 10.26 & -0.53 \\
\hline $\mathrm{Na}_{2}\left(\mathrm{e}^{0}\right)-\mathrm{tTt} \rightarrow \mathrm{Na}_{2}+\mathrm{DME}$ & 7.33 & 9.49 & 6.81 & 9.17 & -0.51 & 6.81 & 9.17 & -0.51 \\
\hline
\end{tabular}

${ }^{a}$ Adiabatic equilibrium dissociation energy with respect to the most stable conformer of DME, tTt. ${ }^{b}$ Zero-point energy correction with respect to the most stable conformer of DME, tTt. ${ }^{c}$ Adiabatic equilibrium dissociation energy with respect to its own conformation state. ${ }^{d}$ Zero-point energy correction with respect to its own conformation state. ${ }^{e}$ DME: 1,2-dimethoxyethane.

TABLE 4: Theoretical Dissociation Energies (kcal/mol) of $\mathrm{Na}^{+}(\mathrm{DME})$ and $\mathrm{Na}_{2}{ }^{+}(\mathrm{DME})(\mathrm{MP2}(\mathrm{fu}) / 6-311+\mathrm{G}(\mathrm{d}, \mathrm{p}) / / \mathrm{Mp} 2(\mathrm{fu}) /$ 6-31G*)

\begin{tabular}{|c|c|c|c|c|c|c|c|c|}
\hline & \multicolumn{2}{|c|}{ sudden } & \multicolumn{6}{|c|}{ adiabatic } \\
\hline & $\mathrm{HF}$ & MP2 & $\mathrm{HF}^{a}$ & $\mathrm{MP}^{a}$ & $\mathrm{ZPEC}^{b}$ & $\mathrm{HF}^{c}$ & $\mathrm{MP} 2^{c}$ & $\mathrm{ZPEC}^{d}$ \\
\hline $\mathrm{Na}^{+}\left(\mathrm{g}^{\prime}\right)-\mathrm{tGt} \rightarrow \mathrm{Na}^{+}+\mathrm{DME}^{e}$ & 48.42 & 47.17 & 43.26 & 44.25 & -1.29 & 44.59 & 44.53 & -1.33 \\
\hline $\mathrm{Na}^{+}\left(\mathrm{g}^{\prime}\right)-\mathrm{tGg} \rightarrow \mathrm{Na}^{+}+\mathrm{DME}$ & 48.16 & 47.03 & 41.65 & 42.69 & -1.19 & 45.27 & 44.42 & -1.07 \\
\hline $\mathrm{Na}^{+}(\mathrm{e})-\mathrm{g}^{\prime} \mathrm{Tt} \rightarrow \mathrm{Na}^{+}+\mathrm{DME}$ & 29.29 & 28.55 & 25.13 & 25.86 & -0.70 & 27.20 & 27.41 & -0.66 \\
\hline $\mathrm{Na}^{+}\left(\mathrm{e}^{0}\right)-\mathrm{tTt} \rightarrow \mathrm{Na}^{+}+\mathrm{DME}$ & 26.95 & 27.04 & 25.42 & 25.95 & -0.58 & 25.42 & 25.95 & -0.58 \\
\hline $\mathrm{Na}^{+}\left(\mathrm{e}^{0}\right)-\mathrm{tTg}^{\prime} \rightarrow \mathrm{Na}^{+}+\mathrm{DME}$ & 26.98 & 26.93 & 23.39 & 24.34 & -0.61 & 25.47 & 25.89 & -0.56 \\
\hline $\mathrm{Na}_{2}^{+}\left(\mathrm{g}^{\prime}\right)-\mathrm{tGt} \rightarrow \mathrm{Na}_{2}^{+}+\mathrm{DME}$ & 37.78 & 37.09 & 32.78 & 34.67 & -1.12 & 34.11 & 34.96 & -1.23 \\
\hline $\mathrm{Na}_{2}{ }^{+}\left(\mathrm{g}^{\prime}\right)-\mathrm{tGg} \rightarrow \mathrm{Na}_{2}^{+}+\mathrm{DME}$ & 35.82 & 36.08 & 31.07 & 33.09 & -1.12 & 34.69 & 34.82 & -0.99 \\
\hline $\mathrm{Na}_{2}^{+}\left(\mathrm{g}^{\prime}\right)-\mathrm{tTt} \rightarrow \mathrm{Na}_{2}^{+}+\mathrm{DME}$ & 20.22 & 20.88 & 19.11 & 20.13 & -0.56 & 19.11 & 20.13 & -0.56 \\
\hline $\mathrm{Na}_{2}^{+}-\mathrm{tGt} \rightarrow \mathrm{Na}+\mathrm{Na}^{+}(\mathrm{DME})$ & 11.45 & 12.54 & 11.26 & 12.34 & -0.07 & 11.26 & 12.34 & -0.07 \\
\hline $\mathrm{Na}_{2}{ }^{+}-\mathrm{tGg} \rightarrow \mathrm{Na}+\mathrm{Na}^{+}(\mathrm{DME})$ & 11.40 & 12.49 & 9.55 & 10.76 & 0.00 & 11.16 & 12.32 & -0.10 \\
\hline
\end{tabular}

${ }^{a}$ Adiabatic equilibrium dissociation energy with respect to the most stable conformer of DME, tTt or, in the case of Na ${ }^{+}(\mathrm{DME})$, Na ${ }^{+}\left(\mathrm{g}^{\prime}\right)-\mathrm{tGt}$ ${ }^{b}$ Zero-point energy correction with respect to the most stable conformer of DME, tTt. ${ }^{c}$ Adiabatic equilibrium dissociation energy with respect to its own conformation state. ${ }^{d}$ Zero-point energy correction with respect to its own conformation state. ${ }^{e}$ DME: 1,2 -dimethoxyethane.

than $\mathrm{g}^{\prime}-\mathrm{tGg}$. This follows the same stability trend of the free conformer state of DME, i.e., tGt conformer is more stable than $\mathrm{tGg}$ conformer. The same trend is also observed among the conformers with internal electrostatic attractive interaction and also among the straight-chain form. Similar to the case of the sudden dissociation energies, there is also extra stability in the adiabatic bond dissociation energies for the conformers with $1,5 \mathrm{CH}_{3} / \mathrm{O}$ interactions if the energy differences among the free DME conformers are taken into account. The cooperative stabilization between the sodium bonding and the internal electrostatic attractive interaction is apparently in operation. Similar trends are also observed in the corresponding disodium complex.

The last three columns of Table 3 list the adiabatic equilibrium dissociation energy of the neutral complexes and the zeropoint energy corrections with respect to the initial conformer state. They are not much different from their corresponding sudden dissociation energies. This is consistent with the fact that the sodium bonding is still too weak to induce appreciable geometric changes in the bonding formation or breaking.

Overall, for the neutral sodium complexes, the bonding energies of the disodium complexes are stronger than those of the corresponding monosodium systems. Similar results were also reported for the potassium-ethanediamine systems. ${ }^{10}$ These are attributed to the larger electric polarizabilities of the diatomic sodium or potassium molecules than those of the corresponding atomic species. The electric dipole-induced dipole interaction has been recognized as an important stabilization factor for this type of bonding. ${ }^{8-10}$

Table 4 lists the bond dissociation energies of the ionic sodium complexes. The major interaction force is the electrostatic interactions between the sodium ion and the polar DME molecule, and therefore, it is expected to be much stronger than the sodium bonding of their corresponding neutral systems. These complexes also show much larger bond energy separations between the monodentate and bidentate conformers. Contrary to their neutral species, the bond dissociation energies of the disodium complexes are less than those of the corresponding monosodium systems by about $10 \mathrm{kcal} / \mathrm{mol}$. Apparently, the delocalization of the positive charge over the sodium molecule weakens the electrostatic interaction of the complexes.

The last two rows of Table 4 show that the bond dissociation energies of the two most stable disodium ionic complexes dissociated into one sodium atom and the most stable monosodium ionic complex are 12.34 and $10.76 \mathrm{kcal} / \mathrm{mol}$, respectively. The average adiabatic ionization potential of the second conformation group of the disodium complexes, which are shown in the last three rows of Table 2, is $3.79 \mathrm{eV}$. Its corresponding average vertical ionization potential is $4.42 \mathrm{eV}$. Their energy difference of $0.63 \mathrm{eV}(14.6 \mathrm{kcal} / \mathrm{mol})$ is already larger than the above largest monosodium ion dissociation energy of $12.3 \mathrm{kcal} / \mathrm{mol}$ even before counting in the internal thermal energy. Energetically, the second conformation group of the disodium complexes would decompose into monosodium ions after the photoionization process as discussed in the previous subsection. 
Experimentally, the enthalpy of dissociation for $\mathrm{Na}^{+}(\mathrm{DME})$ has been measured to be $47.2 \mathrm{kcal} / \mathrm{mol}$ over a temperature range 660-700 K. ${ }^{34,35}$ One may approximate the measured bond dissociation enthalpy as being entirely due to the bond dissociation of the most stable conformer of $\mathrm{Na}^{+}(\mathrm{DME})$ into $\mathrm{Na}^{+}$ and the most stable conformer of DME. With the theoretical harmonic vibrational frequencies for these states, the thermal energy correction for the bond dissociation process is calculated to be $-0.40 \mathrm{kcal} / \mathrm{mol}$ at $680 \mathrm{~K}$. The experimental bond dissociation energy $D_{0}$ of $\mathrm{Na}^{+}(\mathrm{DME})$ becomes $46.8 \mathrm{kcal} / \mathrm{mol}$. The theoretical bond dissociation energies of the sodium complexes in any conformation could be obtained by adding the zero-point energy corrections to the corresponding equilibrium bond dissociation energies listed in Tables 3 and 4 . For the most stable conformer $\mathrm{Na}^{+}(\mathrm{tGt})$, the theoretical bond dissociation energy is $43.0 \mathrm{kcal} / \mathrm{mol}$. The experimental bond dissociation energy is $3.8 \mathrm{kcal} / \mathrm{mol}$ higher than the theoretical value.

Taking the experimental bond dissociation energy to be accurate, one may estimate the bond dissociation energy of the corresponding neutral conformer by the following relation:

$$
\begin{aligned}
D_{0}(\mathrm{Na}(\mathrm{DME}))= & \\
& \mathrm{IP}(\mathrm{Na}(\mathrm{DME}))+D_{0}\left(\mathrm{Na}^{+}(\mathrm{DME})\right)-\mathrm{IP}(\mathrm{Na})
\end{aligned}
$$

in which the experimental ionization potential of $\mathrm{Na}$ is 5.139 $\mathrm{eV}$, the experimental bond dissociation energy of $\mathrm{Na}^{+}(\mathrm{DME})$ is $46.8 \mathrm{kcal} / \mathrm{mol}$, and the experimental adiabatic ionization potential is approximated by the present experimental threshold potential of $3.80 \mathrm{eV}$. One reaches an experimental bond dissociation energy of $15.9 \mathrm{kcal} / \mathrm{mol}$ for $\mathrm{Na}(\mathrm{DME})$. Since the experimental ionization threshold potential may overestimate the adiabatic ionization potential by $0.11 \mathrm{eV}-$ the energy difference between the theoretical vertical and adiabatic ionization potentials for the most stable neutral conformer $\mathrm{g}^{\prime}-\mathrm{tGt}-$ the above experimental bond dissociation may overestimate the bond dissociation by $2.5 \mathrm{kcal} / \mathrm{mol}$. Taking this factor into account, one obtains a final $13.4 \mathrm{kcal} / \mathrm{mol}$ for the experimental bond energy of the neutral conformer $\mathrm{g}^{\prime}-\mathrm{tGt}$. Compared with the corresponding theoretical value of $8.8 \mathrm{kcal} / \mathrm{mol}$, the agreement is not satisfactory. It is known that the local electric dipole strength of the ether group is comparable to that of the amino group but weaker than that of the hydroxyl group if one compares the dipole moments of dimethyl ether, methylamine, and methanol and that the electric polarizability of the potassium atom is larger than that of the sodium atom. Based on the dipole-induced dipole model, it is expected that for a given alkali metal atom the bond strengths of the DME complexes are comparable to those of ethylenediamine but weaker than those of the 1,2-ethanediol and that the bond strengths of the potassium complexes are generally slightly stronger than those of the corresponding sodium complexes. ${ }^{8-10,36}$ The present 13.4 $\mathrm{kcal} / \mathrm{mol}$ sodium-DME bond energy are in the high-value range of the expected bond energies among the other similar class of the complexes.

If one starts from the theoretical bond dissociation energy of $\mathrm{Na}^{+}(\mathrm{DME})$ of $43.0 \mathrm{kcal} / \mathrm{mol}$ and follows the above procedure, one obtains the bond dissociation of the neutral conformer to be $9.6 \mathrm{kcal} / \mathrm{mol}$, a value in good agreement with the theoretical prediction. In short, the present experimental ionization potential is internally consistent with the theoretical results. The reported experimental bond dissociation energy of $\mathrm{Na}^{+}(\mathrm{DME})$ is about $3-4 \mathrm{kcal} / \mathrm{mol}$ too large to conform to the present experimental photoionization measurements and the theoretical data of the sodium-DME complex. Three factors may contribute to the discrepancy. First, in the enthalpy measurements, the enthalpy contributions of the higher-energy conformers of free DME under the relatively high-temperature experimental condition may also be significant, a factor neglected in the above calculation. Second, there are contributions of the second and third higher-energy cyclic conformers in the present photoionization experiments. These conformers have higher vertical-to-adiabatic ionization energy differences than that of the most stable cyclic conformer, and therefore, a higher-energy correction would be needed in the above bond dissociation energy calculation. Third, in the enthalpy measurements, the evidence of the decomposition of some DME at the experimental temperatures might also lead to a higher measured value for the dissociation enthalpy of $\mathrm{Na}^{+}(\mathrm{DME}){ }^{35}$ As a final note, for the disodium complexes, there were no other related reports available in the literature.

\section{Conclusions}

The conformation-dependent photoionization potentials and bond dissociation energies of the molecular complexes$\mathrm{Na}$ (DME), $\mathrm{Na}_{2}$ (DME), $\mathrm{Na}^{+}(\mathrm{DME})$, and $\mathrm{Na}_{2}{ }^{+}$(DME)-were studied by the photoionization and molecular orbital methods. The following conclusions could be drawn from the present studies.

(1) With the help of the molecular orbital calculations, the photoionization efficiency spectra of $\mathrm{Na}(\mathrm{DME})$ and $\mathrm{Na}_{2}$ (DME) could be attributed to the contributions of various conformation groups. The threshold ionization energies of the most stable conformation groups were assigned and determined.

(2) The conformation-dependent bonding strengths for both the neutral and ionic sodium complexes were calculated by the molecular orbital methods and classified according to the number of the sodium bonds and also the $1,5 \mathrm{CH}_{3} / \mathrm{O}$ electrostatic interaction. The related experimental results, which include the dissociation enthalpy of $\mathrm{Na}^{+}(\mathrm{DME})$ and the threshold ionization potentials of $\mathrm{Na}(\mathrm{DME})$, were assessed.

Acknowledgment. The financial support of this work by the National Science Council, Republic of China, is gratefully acknowledged.

Supporting Information Available: Tables of the geometric parameters and harmonic vibrational frequencies of all the stable conformers of $\mathrm{Na}(\mathrm{DME}), \mathrm{Na}^{+}(\mathrm{DME}), \mathrm{Na}_{2}(\mathrm{DME})$, and $\mathrm{Na}_{2}{ }^{+}(\mathrm{DME})$ discussed in this report (15 pages). Ordering information is given on any current masthead page.

\section{References and Notes}

(1) Trenary, M.; Schaefer, H. F., III; Kollman, P. A. J. Am. Chem. Soc. 1977, 99, 3885.

(2) Trenary, M.; Schaefer, H. F., III; Kollman, P. A. J. Chem. Phys. 1978, 68, 4047.

(3) Curtiss, L. A.; Frurip, D. J. Chem. Phys. Lett. 1980, 75, 69

(4) Bentley, J.; Carmichael, I. J. Phys. Chem. 1981, 85, 3821.

(5) Bentley, J. J. Am. Chem. Soc. 1982, 104, 2754.

(6) Broughton, J. Q.; Bagus, P. S. J. Chem. Phys. 1982, 77, 3627.

(7) Curtiss, L. A.; Pople, J. A. J. Chem. Phys. 1985, 82, 4230.

(8) Curtiss, L. A.; Kraka, E.; Gauss, J.; Cremer, D. J. Phys. Chem. 1987, 91, 1080. 335

(9) Hsiao, Y.-W.; Chang, K.-M.; Su, T.-M. Chem. Phys. 1992, 162,

(10) Liau, Y.-H.; Su, T.-M. J. Am. Chem. Soc. 1992, 114, 9169.

(11) Schulz, C. P.; Haugstatter, R.; Tittes, H. U.; Hertel, I. V. Phys. Rev. Lett. 1986, 57, 1703.

(12) Schulz, C. P.; Haugstatter, R.; Tittes, H. U.; Hertel, I. V. Z. Phys. D: At. Mol. Clusters 1988, 10, 279.

(13) Kuan, T.-C.; Jiang, R. C.; Su, T.-M. J. Chem. Phys. 1990, 92, 2553.

(14) Hertel, I. V.; Huglin, C.; Nitsch, C.; Schulz, C. P. Phys. Rev. Lett. $1991,67,1767$.

(15) Hsu, C.-L.; Yeh, T.-S.; Su, T.-M. J. Phys. Chem. 1994, 98, 8876. 
(16) Barzaghi, M.; Gamba, A.; Morosi, G. J. Mol. Struct.: THEOCHEM 1988, 170,69

(17) Murcko, M. A.; DiPaola, R. A. J. Am. Chem. Soc. 1992, 114, 10010.

(18) Bressanini, D.; Gamba, A.; Morosi, G. J. Phys. Chem. 1990, 94, 4299.

(19) Tsuzuki, S.; Uchimaru, T.; Tanabe, K.; Hirano, T. J. Phys. Chem. 1993, 97, 1346.

(20) Liu, H. ; Müller-Plathe, F. F.; van Gunsteren, W. F. J. Chem. Phys. 1993, 102, 1722 .

(21) Ogawa, Y.; Ohta, M.; Sakakibara, M.; Matsuura, H.; Harada, I. Shimanouchi, T. Bull. Chem. Soc. Jpn. 1977, 50, 650.

(22) Astrup, F. E. Acta Chem. Scand. 1977, A33, 655.

(23) Inomata, K.; Abe, A. J. Phys. Chem. 1992, 96, 7934.

(24) Yoshida, H.; Kaneko; Matsuura, H.; Ogawa, Y.; Tasumi, M. Chem. Phys. Lett. 1992, 196, 601.

(25) Jaffe, R. L.; Smith, G. D.; Yoon, D. Y. J. Phys. Chem. 1993, 97, 12745 .

(26) Smith, G. D.; Jaffe, R. L.; Yoon, D. Y. J. Phys. Chem. 1993, 97, 12752 .

(27) Smith, G. D.; Jaffe, R. L.; Yoon, D. Y. J. Am. Chem. Soc. 1995, $117,530$.

(28) Frisch, M. J.; Trucks, G. W.; Schlegel, H. B.; Gill, P. M. W.; Johnson, B. G.; Robb, M. A.; Cheeseman, J. R.; Keith, T. A.; Petersson,
G. A.; Montgomery, J. A.; Raghavachari, K.; Al-Laham, M. A.; Zakrzewski, V. G.; Ortiz, J. V.; Foresman, J. B.; Cioslowski, J.; Stefanov, B. B.; Nanayakkara, A.; Challacombe, M.; Peng, C. Y.; Ayala, P. Y.; Chen, W.; Wong, M. W.; Andres, J. L.; Replogle, E. S.; Gomperts, R.; Martin, R. L.; Fox, D. J.; Binkley, J. S.; Defrees, D. J.; Baker, J.; Stewart, J. P.; HeadGordon, M.; Gonzalez, C.; Pople, J. A. Gaussian 94, Revision B.2; Gaussian, Inc.: Pittsburgh, PA, 1995.

(29) Radom, L.; Lathan, W. A. ; Hehre, W. J.; Pople, J. A. J. Am. Chem. Soc. 1973, 95, 693.

(30) Yeh, T.-S.; Chang, Y.-P.; Su, T.-M.; Chao, I. J. Phys. Chem. 1994, $98,8921$.

(31) Moore, C. E. Atomic Energy Levels; NSRD-NBS Vol. 1; US GPO: Washington, DC, 1971; p 89.

(32) Martin, S.; Chevaleyre, J.; Valignat, S.; Perrot, J.; Broyer, M.; Cabaud, B.; Hoareau, A. Chem. Phys. Lett. 1982, 87, 235.

(33) Becke, A. D. J. Chem. Phys. 1993, 98, 5648.

(34) Castleman, A. W., Jr.; Peterson, K. I.; Upschulte, B. L.; Schelling, F. J. Int. J. Mass Spectrom. Ion Phys. 1983, 47, 203.

(35) Peterson, K. I. Ph.D. Thesis, The University of Colorado, Boulder, Colorado, 1982.

(36) Yeh, T.-S.; Su, T.-M. Unpublished results. 